\title{
自然科学基金项目进展专栏
}

评 述

\section{血小板蛋白质组学及其在血瘀证与活血化瘀中药 研究中的探索应用}

\author{
刘玥 ${ }^{(1)}$, 殷惠军 ${ }^{(1) *}$, 陈可冀 ${ }^{(1) * *}$ \\ (1) 中国中医科学院西苑医院心血管病中心, 北京 100091; \\ (2) 中国中医科学院心血管病研究所, 北京 100091 \\ * 联系人, E-mail: huijunyin@aliyun.com; kjchenvip@ 163.com
}

收稿日期: 2013-05-02; 接受日期: 2013-06-25

国家自然科学基金重点项目(批准号: 81030063)和国家自然科学基金(批准号: 30901949, 81073086, 81102845)资助

\begin{abstract}
摘要 寻找新的安全有效的抗血小板药物或能改善抗血小板药物抵抗的方法, 成为心脑 血管疾病防治领域的国际性关注点. 不断发展成熟的血小板蛋白质组学技术为深入开展高 质量的血小板病生理特征研究及新药开发提供了良好的技术平台. 血瘀证实质与活血化瘀 中药的科学化研究一直是传统中医药学和中西医结合研究中最为活跃的领域之一. 临床治 疗中医血瘀证的主要药物——活血化瘀中药不少具有抗血小板及抗动脉籿样硬化的临床作 用, 但缺乏明确的作用靶点. 冠心病、血瘀证及血小板功能状态三者之间存在密切关系. 采 用血小板蛋白质组学技术, 研究冠心病血瘀证活化血小板差异表达的蛋白质及分析其功能, 不仅对阐明活血化瘀抗血小板中药的作用机制具有重要意义, 而且可为此类新药开发及临 床评价体系的建立奠定基础. 本研究对近年来血小板蛋白质组学及其在血瘀证实质及活血 化瘀中药研究中的探索应用进行评述.

关键词 血小板蛋白质组 活血化瘀 血瘀证 冠心病 中医药
\end{abstract}

血小板是从骨髓成熟的巨核细胞胞质裂解继而 脱落下来的具有生物活性的微小胞质, 在止血、炎症 反应、血栓形成及器官移植排斥等病理生理过程中均 扮演了极其重要的角色. 血小板活化及继发炎症反 应介导的动脉粥样硬化易损斑块破裂和急性血栓形 成是急性血管事件发生的重要病理生理基础. 抗血 小板药物的临床应用成为心脑血管疾病治疗学领域 的里程碑事件 ${ }^{[1]}$, 目前阿司匹林及氯吡格雷等已成为 预防和治疗动脉强样硬化血管性疾病的基石药物. 但随着双重甚至三重抗血小板药物应用时间的延长,
出现了血小板反应的多样性, 具体表现为低反应性 和高反应性，前者表现为即使临床及时、足量应用抗 血小板药物但血栓风险仍未降低, 又称为“抗血小板 药物抵抗 ${ }^{[2,3]}$; 后者主要表现为消化系统或神经系统 出血风险的增加. 这些都限制了抗血小板药物的临 床应用, 同时也提示血小板可能还存在其他的活化 通路或途径, 因此寻找新的安全有效的抗血小板药 物或者能改善抗血小板药物抵抗的方法, 成为心脑 血管疾病治疗领域的国际性关注点.

引用格式: 刘玥, 殷惠军, 陈可冀. 血小板蛋白质组学及其在血瘀证与活血化瘀中药研究中的探索应用. 中国科学: 生命科学, 2013,43: 619-625

英文版见: Liu Y, Yin H J, Chen K J. Platelet Proteomics and Its advanced application for the research of activated bloodcirculation herbs of Chinese medicine. Sci China Life Sci, 2013, 56, in press 


\section{1 血小板蛋白质组学}

蛋白质组学从整体水平上研究生物体蛋白质的 组成及其变化规律, 为全面揭示重大疾病发生发展 的分子机制及干预药物的作用靶点提供了全新的研 究思路和策略. 血小板是无核细胞, 但具有丰富的胞 内颗粒, 主要包括 $\alpha$-颗粒、致密体和溶酶体等, 其继 承于巨核细胞的转录本和一些蛋白质合成结构, 使 血小板具备一定的蛋白质合成和修饰功能, 是蛋白 质组学理想的研究对象. 以血小板为基础, 通过鉴定 血小板在静息或活化状态时表达的特殊蛋白质及血 小板代谢的相关信号通路，进而分析血小板蛋白质 在正常与病理状态下的功能变化, 可以丰富对血小 板生物学功能的理解 ${ }^{[4]}$. 近年来不断发展的高通量蛋 白质组学技术为篮选新的抗血小板作用靶点提供了 可能. 荧光差异显示二维凝胶电泳 (two-dimensional fluorescence difference gel electrophoresis , 2-D DIGE) 技术，是进行蛋白质差异表达分析的理想技术平台， 在检测和分析复杂蛋白质样品的蛋白质表达差异或 变化时, 它良好的重现性有利于获得可靠的实验结 果, 克服了传统二维凝胶电泳不同胶之间的不可重 现性和严重的系统变异, 为不同胶或/和生命系统不 同状态之间变化的研究提供了完善的解决方案. 与 2-D DIGE 相衔接的基质辅助激光解析/电离飞行时间 串联质谱(MALDI-TOF-TOF)技术同样具有高灵敏性 与特异性. 血小板蛋白质组学技术目前广泛用于人 类重大疾病如血液病、冠心病等的早期诊断(疾病标 志分子)和新药研发(药物干预靶点)等的研究当中, 其研究设计模式通常分为篮选一鉴定(定性及定量) 一功能分析 3 个阶段 ${ }^{[5 \sim 7]}$.

2012 年发表在国际著名期刊 Blood 上的一项国 外学者的研究采用定量质谱 (quantitative mass spectrometry)技术, 首次全面、定量地建立了人血小 板功能蛋白质组 ${ }^{[8]}$, 鉴定出超过 2500 个磷酸化位点, 共包含有 4000 种血小板蛋白. 同时采用比较蛋白质 组学方法, 在 4 个健康志愿者血样之间鉴定出 1900 种血小板蛋白, 在同一个健康志愿者的 3 份不同血样 之间鉴定出 1500 种血小板蛋白. 同时发现即使存在 一定的正常变异, 总体来说, $85 \%$ 被鉴定出的健康志 愿者血小板蛋白具有恒定性, 该研究为后续血小板 蛋白的功能分析奠定了良好的基础.

\section{2 血小板蛋白质组学与血瘀证实质研究}

蛋白质组学着眼于从不同层次“整体”蛋白质功 能活动的特征来探究机体活动的内在规律, 与传统 中医学整体观及中药多靶点整合调节的特性不谋而 合, 近年来临床医学也逐渐从片段化特征回归到整 体整合模式 ${ }^{[9]}$. 随着中国中医药学与西医药学的碰 撞、发展及两种医药学在真实医疗环境中的相互交 融、渗透, 中西医结合医学应运而生, 成为我国独具 特色的医疗体系. 目前中西医结合医学界最为普遍 采取的是西医辨病与中医辨证论治相结合的现代“病 证结合”研究模式, 这也是中西医结合医学的重要发 展方向之一 ${ }^{[10]}$. 在中医证候理论的指导下, 运用功能 蛋白质组学的方法, 研究典型中医证候差异蛋白质 组的表达, 分析与某一中医证候形成相关的所有蛋 白质及其特征，并进行功能研究，在整体蛋白质表达 水平上阐明中医证候的本质，以期为中医证候的科 学化提供实证依据 ${ }^{[11]}$.

中医血疼证存在血小板活化现象，研究表明，冠 心病、血瘀证、血小板功能状态三者之间存在极其密 切的内在联系 ${ }^{[12]}$. 在早期建立血瘀证诊断标准的基 础上, 近年来又建立了冠心病血疼证的诊断标准 ${ }^{[13]}$, 为开展血瘀证实质的系统研究提供了良好的基础. 血小板是冠脉血栓形成的中心环节，激活的血小板 不但参与形成血栓, 还释放多种血管活性物质、细胞 因子和生长因子, 参与动脉秥样硬化的形成和发展. 血疼证与血液循环和微循环障碍、血液高黏滞状态、 血小板活化和黏附聚集、血栓形成等多种病理生理改 变密切相关, 血瘀证是冠心病临床最常见的中医证 型, 既往研究报道冠心病血瘀证存在血小板活化状 态 $^{[14]}$.

本实验组在国内外较早地将血小板蛋白质组学 技术引入中医证候实质和中药作用机制研究，以冠 心病血瘀证与活血化瘀中药为切入点, 在国家自然 科学基金的连续资助下遵循篮选一鉴定一验证一功 能分析的模式开展了一系列创新性探索研究. 前期 采用差异蛋白质组学的研究方法, 借助苂光差异显 示 2-D DIGE 技术及 MALDI-TOF-TOF 技术篮选到冠 心病血瘀证与非血瘀证组有 45 个血小板差异蛋白质 点(图 1) $)^{[15]}$, 又根据每个点的三维图、等电点、分子 质量和在胶上实际位置对应信息匹配程度，确定 23 个点，切胶. 质谱成功鉴定 14 个差异蛋白质(表 1 ), 


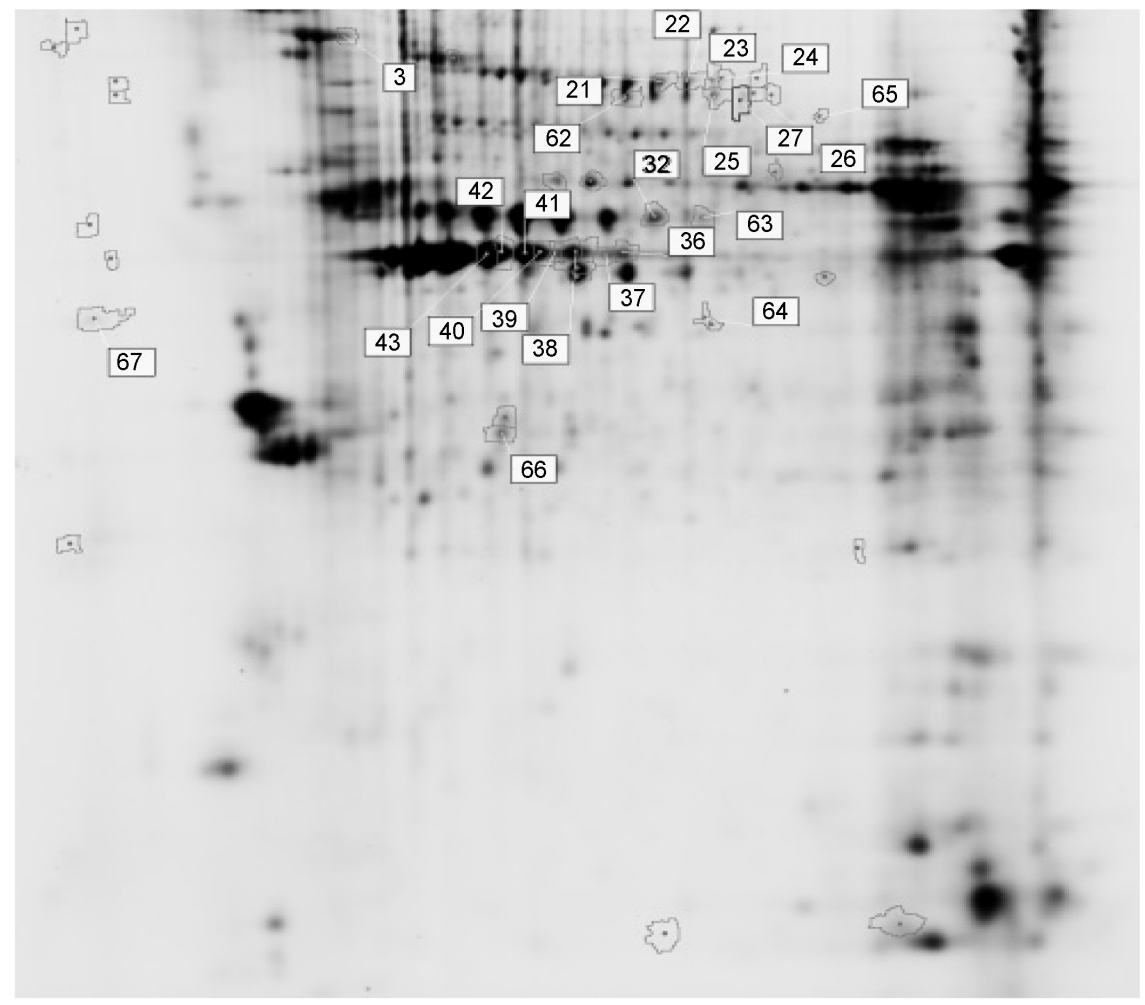

图 1 冠心病血瘀证组与非血瘀证组患者差异表达蛋白质胶图(经 DeCyder 2D ${ }^{\mathrm{TM}}$ 分析后) ${ }^{[15]}$

表 1 冠心病血瘀证与非血瘀证组血小板差异蛋白质搜库鉴定成功结果 ${ }^{[15]}$ a)

\begin{tabular}{|c|c|c|c|c|c|c|c|c|}
\hline 蛋白质编号 & 蛋白质名称 & $\begin{array}{l}\text { 蛋白质相对 } \\
\text { 分子质量 }\end{array}$ & $\begin{array}{l}\text { 蛋白质 } \\
\text { 等电点 }\end{array}$ & $\begin{array}{l}\text { 蛋白质 } \\
\text { 评分 }\end{array}$ & $\begin{array}{l}\text { 蛋白质评 } \\
\text { 分置信区 } \\
\text { 间 }(\%)\end{array}$ & $\begin{array}{l}\text { 总离子 } \\
\text { 评分 }\end{array}$ & $\begin{array}{l}\text { 总离子评 } \\
\text { 分置信区 } \\
\text { 间 }(\%)\end{array}$ & $\begin{array}{c}\text { 差异蛋 } \\
\text { 白质点 } \\
\text { 在胶图 } \\
\text { 中的编 } \\
\text { 号 }\end{array}$ \\
\hline IPI00218628 & $\begin{array}{l}\text { Gene_Symbol=ITGA2B Isoform } 2 \text { of Integrin } \\
\text { alpha-IIb }\end{array}$ & 109518.5 & 5.17 & 751 & 100 & 651 & 100 & 3 \\
\hline IPI00877792 & Gene_Symbol=FGG 50 kDa protein & 50290.4 & 5.71 & 141 & 100 & 62 & 99.929 & 32 \\
\hline IPI00739539 & Gene_Symbol=A26C1B ANKRD26-like & 121292.7 & 5.86 & 170 & 100 & 143 & 100 & 36 \\
\hline IPI00894365 & Gene_Symbol=ACTB cDNA FLJ52842, & 39200.5 & 5.4 & 321 & 100 & 252 & 100 & 37 \\
\hline IPI00021439 & Gene_Symbol=ACTB Actin, cytoplasmic 1 & 41709.7 & 5.29 & 445 & 100 & 330 & 100 & 38 \\
\hline$\underline{\text { IPI00021439 }}$ & $\begin{array}{l}\text { Tax_Id=9606 Gene_Symbol=ACTB Actin, } \\
\text { cytoplasmic } 1\end{array}$ & 41709.7 & 5.29 & 342 & 100 & 274 & 100 & 39 \\
\hline$\underline{\text { IPI00021439 }}$ & Gene_Symbol=ACTB Actin, cytoplasmic 1 & 41709.7 & 5.29 & 440 & 100 & 379 & 100 & 40 \\
\hline IPI00021440 & Gene_Symbol=ACTG1 Actin, cytoplasmic 2 & 41765.8 & 5.31 & 602 & 100 & 480 & 100 & 41 \\
\hline IPI00021439 & Gene_Symbol=ACTB Actin, cytoplasmic 1 & 41709.7 & 5.29 & 588 & 100 & 478 & 100 & 42 \\
\hline IPI00894365 & $\begin{array}{l}\text { Gene_Symbol=ACTB cDNA FLJ52842, } \\
\text { highly similar to Actin, cytoplasmic } 1 \\
\text { Tax_Id=9606 Gene_Symbol=ACTG1 cDNA }\end{array}$ & 39200.5 & 5.4 & 442 & 100 & 326 & 100 & 43 \\
\hline IPI00794523 & $\begin{array}{l}\text { FLJ } 43573 \text { fis, clone RECTM2001691, highly } \\
\text { similar to Actin, cytop }\end{array}$ & 28193 & 5.2 & 236 & 100 & 189 & 100 & 66 \\
\hline IPI00298497 & Gene_Symbol=FGB Fibrinogen beta chain & 55892.3 & 8.54 & 80 & 99.924 & 59 & 99.867 & 63 \\
\hline IPI00009865 & $\begin{array}{l}\text { Gene_Symbol=KRT10 Keratin, type I } \\
\text { cytoskeletal } 10\end{array}$ & 59474.9 & 5.13 & 141 & 100 & 56 & 99.903 & 23 \\
\hline IPI00796316 & $\begin{array}{l}\text { Gene_Symbol=GSN cDNA FLJ53327, highly } \\
\text { similar to Gelsolin }\end{array}$ & 77741.1 & 5.47 & 83 & 99.958 & 40 & 95.153 & 62 \\
\hline
\end{tabular}

a) 蛋白质编号斜体加下画线的是冗余 
去除 4 个圥余, 找到 10 个有可靠数据支撑的血小板 差异表达功能蛋白质点. 从中可以发现除血小板膜 蛋白外，还包括了许多血小板骨架蛋白. 血小板活化 不但可引起其膜蛋白的改变, 亦可导致一系列形态 学变化, 即从无黏性的、盘状的循环血小板变成有黏 性的、有突起的血小板胶状物, 而这种形态学变化依 赖于血小板细胞骨架蛋白的调控. 通过文献调研, 本 研究选择其中功能相对清楚的血小板骨架蛋白凝溶 胶蛋白(Gelsolin)进行了规模临床验证, 结果证实冠 心病血瘀证患者血小板 Gelsolin 的含量较冠心病非 血瘀证患者及健康对照人群显著升高 ${ }^{[16]}$, 提示 Gelsolin 与冠心病血疙证的发生相关, 推测血小板骨 架蛋白含量异常在冠心病血瘀证的发生中发展扮演 了重要角色. 同时又将血小板 Gelsolin 含量与冠心病 不同分型(稳定型冠心病、急性冠脉综合征)之间的相 关性开展了临床研究, 结果表明其水平与急性冠脉 综合征(acute coronary syndrome, ACS)的发生高度正 相关 ${ }^{[17]}$.

近年来 Gelsolin 在心血管疾病发生发展中的作 用越来越得到国内外学术界的重视, 研究发现其可 能在冠心病、心律失常、心肌梗死后心室重构等心血 管疾病中均发挥了重要作用 ${ }^{[18,19]}$. Gelsolin 是细胞骨 架的重要组成部分, 其分泌受到钙离子浓度的调节, 当胞内钻离子浓度升高时可激活血小板 Gelsolin 的 分泌. 梗死后心肌组织释放大量肌动蛋白微丝 (F-actin)入血对内皮细胞及微血管产生毒害作用, 而 血浆中存在肌动蛋白清除系统 (extracellular actin scavenger systern, EASS) ${ }^{[20]}$, 其中血浆 Gelsolin 能够 切割过多的 F-actin, 使其解聚成肌动蛋白单体 (G-actin), 维生素 D 结合蛋白(vitamin D binding protein, VDBP) 能够和 G-actin 结合形成复合物, 通过 肝脏内皮网状系统被清除体外而阻止这种细胞毒效 应. 研究发现 ${ }^{[16]}$, 冠心病血瘀证血小板活化过程中, 细胞骨架蛋白含量也出现了不同程度的变化, 集中 体现在血小板 Gelsolin 和其靶蛋白 F-actin 的异常变 化上, 推测血浆 Gelsolin 含量降低与其因切割、清除 F-actin 而大量消耗有关, 这种消耗引起血小板上的 Gelsolin 病理性重组而含量增加, 同时引起血小板钙 离子内流增加, 导致血小板变形能力增强而参与冠 心病血疼证的形成, 因此血小板 Gelsolin 可能是冠心 病血瘀证的特异性分子靶标之一.

\section{3 血小板蛋白质组学与活血化瘀中药研究}

活血化疼方药是中医临床治疗血瘀证的主要药 物, 50 多年来, 国内学者在继承传统中医理论思维的 基础上, 从理论、实验及临床等方面对其进行了较为 深入的现代科学化研究, 取得了一系列重大进展 ${ }^{[21]}$, 系统阐明了活血化瘀中药的基本治疗规律与作用原 理, 活血化疼的治疗理念在国内外医学界已得到广 泛共识. 中西医学在对动脉樕样硬化易损斑块的防 治方面，有着稳定病变、“通其血脉”的共同看法，东 西方这种理念上的一致性，使得应用传统活血化瘀 方药对降低心血管风险可能性的探索具有实际意义 ${ }^{[22]}$. 对活血化瘀方药开展的系列研究表明，丹参、丹皮、 当归、赤芳、蒲黄、川芎、三七等活血化瘀中药和血 府逐瘀汤、冠心 II 号、桃红四物汤等活血化瘀复方均 有抗血小板和抗炎作用，并已发现的活血化瘀中药 抗血小板有效成分包括黄酮类(如暮根素、红花黄色 素)、生物碱类(如川芎总碱、川芎嗪等)、萜类(如丹 参酮、三七总㿝苷等)、有机酸类(如丹参素、丹酚酸、 木脂素类等)等, 但其研究多局限于血小板聚集率、血 小板受体等表面现象的药理学观察, 对血小板活化 后蛋白质表达的影响涉及很少, 且作用靶点及信号 通路研究不够明确和深入 ${ }^{[23]}$. 采用血小板蛋白质组 学研究方法，可以为基于中医“方证对应”的活血化 瘀中药及复方药理作用机制的阐明提供许多潜在的 作用靶点 ${ }^{[24]}$.

\section{1 赤莐与川芦}

赤药和川芎均是传统的活血化瘀中药, 药理研 究表明, 均有较好的抗血小板聚集及抗血栓形成的 作用. 芦苟胶囊(川芎、赤苟的有效组分川芦总酚、赤 芬总苷配伍，XSC)是在传统活血化瘀名方血府逐㾉 汤的研究基础上, 结合现代药理研究, 采用其主要药 物川芦、赤莐的有效组分组成的 II 类中药新药复方制 剂. 实验研究发现, XSC 具有扩张冠状动脉、改善心 肌缺血缺氧、抑制血小板聚集及平滑肌细胞增殖、抗 脂质过氧化及保护血管内皮细胞、促进缺血心肌血管

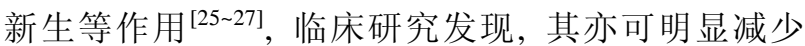
冠心病介入治疗后患者再狭窄的发生率 ${ }^{[28,29]}$. 血小 板活化是动脉粥样硬化形成、不稳定斑块破裂及血栓 形成的关键病理环节，而 XSC 对血小板功能的调节 
作用及其作用靶点的深入研究将为临床抗血小板药 物治疗提供新的思路.

研究如前所述, 冠心病血瘀证血小板活化状态 下循环中聚集的大量 F-actin 在消耗血浆中 Gelsolin 的同时是否可以直接刺激血小板分泌 Gelsolin 而导 致血小板 Gelsolin 含量升高? 血小板 Gelsolin 是否是 活血化瘀方药抗血小板及血栓形成的有效分子靶点? 针对这些推测, 又以 Gelsolin 为研究对象分别建立了 离体血小板活化实验体系及在体心肌梗死大鼠模型. 离体研究发现, 中高浓度的 F-actin 体外能够直接激 活血小板导致其聚集、活化, 且能够刺激血小板大量 分泌 Gelsolin. 赤药、川芎有效成分苟药苷、川芎嗪 体外联合应用对聚集、活化后的血小板具有明显抑制 作用, 同时能够明显降低活化后血小板 Gelsolin 的含 量 ${ }^{[30]}$. 体内研究发现结扎前降支的心肌梗死模型大 鼠贫血小板血浆中 Gelsolin 含量显著降低而富血小 板血浆中 Gelsolin 含量明显增高, 伴随有血浆 F-actin 和钙离子浓度显著上升. 芎芳胶囊能明显降低心肌梗 死大鼠血小板 Gelsolin 水平, 增强血浆肌动蛋白清除 系统活性，抑制血小板活化，同时具有一定的钙怙抗 效应 $^{[31]}$. 通过以上一系列研究证实, 血小板骨架蛋白 Gelsolin 可能是活血化瘀中药的一个有效作用靶点.

\section{2 三七与丹参}

三七药用已有超过 500 年的历史(据《本草纲目》 记载), 其中主要含有㿝苷、黄酮、蛋白质氨基酸及非 蛋白质氨基酸等, 其中皇苷类成分是三七活血化瘀 功效应用的物质基础, 且含量较其他组分高. 有研究 ${ }^{[32]}$ 建立了三七水溶性成分总提物三七总㿝苷温浴的大 鼠血小板和正常大鼠血小板的二维电泳图谱, 识别 并鉴定了两不同组别血小板蛋白质之间的 12 个差异 蛋白, 这些差异蛋白质中有 3 个表达上调, 9 个表达 下调, 说明三七总㿝苷在抗血小板聚集过程中对部 分蛋白质的表达起促进作用, 而另外一些蛋白质的 表达则起抑制作用. 鉴定的 12 个差异表达蛋白按其 生物学功能大致可分为血小板激活相关蛋白(Grb2 蛋 白、凝血酶敏感蛋白 1)、氧化应激相关蛋白(硫氧还 蛋白、核糖核酸酶抑制因子等)、细胞骨架蛋白(肌球 蛋白调节轻链 9、微管蛋白 $\alpha 6$ ) 及其他蛋白(层黏连蛋 白受体 1).

丹参始载于《神农本草经》, 历代本草多有收载, 具有良好的活血化瘀功效, 其主要化学成分主要为
脂溶性二萜醌类(如丹参酮 I, II A, II B 等)和水溶 性酚酸类化合物(如丹酚酸 B、丹参素等). 有学者建 立了丹酚酸 $\mathrm{B}$ 温浴的大鼠血小板和正常大鼠血小板 的二维电泳图谱, 识别并鉴定了两不同组别血小板 蛋白质之间的 20 个差异蛋白, 鉴定的 20 个差异表达 蛋白按其生物学功能大致可分为血小板聚集与血液 凝固相关蛋白(PAF 乙酰水解酶 Ib- $\beta$, FGL2 凝血酶原 酶等)、细胞跨膜信号转导相关蛋白质(电压依赖性钾 离子通道, 14-3-3e, Copine $\mathrm{J}$ 等)、与物质能量代谢相 关的蛋白质(醛缩酶 A)、细胞骨架蛋白(冠蛋白、原肌 球蛋白等)及其他蛋白质(热休克蛋白 70-2、硫氧还原 蛋白过氧化物酶 2、锌指蛋白等 $)^{[33]}$.

\section{4 思考与展望}

中西医结合医学作为全球补充与替代医学 (complementary and alternative medicine)的重要组成 部分, 涌现出的诸如三氧化二砷治疗白血病、青蒿素 及其衍生物治疗㾏疾, 以及活血化瘀中药治疗心血 管疾病等一系列原创性成果越来越受到国内外医学 界的重视和认同 ${ }^{[34]}$. 传统中医药学的发展需要接受 现代科学的洗礼 ${ }^{[35]}$, 利用现代科学技术对中医药现 有的临床治疗学经验或药物进行探索研究是中西医 结合医学发展的重要方向之一.

中医证候是中医药学理论体系的核心, 对其实 质进行科学化阐述不仅是中医药学发展的要求, 也 是中医药现代化的必然选择. 血小板在动脉称样硬 化血栓形成中扮演了关键角色, 而后者又是多种心 脑血管疾病共同的病理基础, 血小板蛋白质组学的 出现及不断发展完善为基于“病证结合”深入探讨中 医证候实质提供了良好的技术平台. 通过对同一疾 病不同中医证候患者的血浆样品中血小板蛋白质表 达谱的比较分析, 可以篎选出相关证候的潜在标志 分子群．同时通过对中医“方证相应”或“方证相关”[36] 的理论指导下的临床方药的干预前后的血小板蛋白 质表达谱的分析, 有助于识别、发现某一治法或方药 的干预靶点, 获得对中药复方作用机制的理解, 同时 也可为抗血小板中药新药笁选及临床疗效评价提供 实证依据. 但需要注意的是, 目前对于重大疾病的血 小板蛋白质组学研究多停留在不同病理生理状态下 血小板蛋白表达谱的篮选和鉴定阶段, 极少涉及对 其功能的后续分析. 目前研究中对血小板低丰度蛋 
白鉴定率不高及如何对获得的海量数据进行合理分 析等, 都是有待解决的问题. 相信未来随着血小板蛋 白质组研究成果的不断丰富及其在血瘀证与活血化
瘀中药研究中的运用, 人们将会获得更多令人惊喜 的新发现和新成果，为降低心脑血管疾病的发病率 和死亡率做出贡献.

\section{参考文献}

1 Michelson A D. Antiplatelet therapies for the treatment of cardiovascular disease. Nat Rev Drug Discov, 2010, 9: 154-169

2 Gergely F, Andrea F, Gabriella P, et al. Clinical importance of aspirin and clopidogrel resistance. World J Cardiol, 2010, 2: 171-186

3 Pena A, Collet J P, Hulot J S. Can we override clopidogrel resistance? Circulation, 2009, 119: 2854-2857

4 杨义玲, 刘文君. 血小板蛋白质组学的研究进展. 中国实验血液学杂志, 2012, 20: 777-781

5 di Michele M, van Geet C, Freson K. Proteomics to unravel platelet-related diseases and identify novel anti-platelet drugs. Curr Med Chem, 2012, 19: $4662-4670$

6 van der Meijden P E, Heemskerk J W. Platelet protein shake as playmaker. Blood, 2012, 120: 2931-2932

7 di Michele M, van Geet C, Freson K. Recent advances in platelet proteomics. Expert Rev Proteomics, 2012, 9: 451-466

8 Burkhart J M, Vaudel M, Gambaryan S, et al. The first comprehensive and quantitative analysis of human platelet protein composition allows the comparative analysis of structural and functional pathways. Blood, 2012, 120: e73-82

9 陈可冀, 刘玥. 临床医学本来就是整体. 医学与哲学, 2013, 3A: 12-13

10 陈可冀. 病证结合治疗观与临床实践. 中国中西医结合杂志, 2011, 31: 1016-1017

11 周红光, 陈海彬, 周学平, 等. 蛋白质组学是中医病机研究的重要技术平台. 中国中西医结合杂志, 2012, 32: 990-993

12 陈可冀, 薛梅, 殷惠军. 血小板活化与冠状动脉挡样硬化性心脏病和血瘀证的关系. 首都医科大学学报, 2008, 29: 266-269

13 付长庚, 高铸烨, 王培利, 等. 冠心病血瘀证诊断标准研究. 中国中西医结合杂志, 2012, 32: 1285-1286

14 Xue M, Chen K J, Yin H J. Relationship between platelet activation related factors and polymorphism of related genes in patients with coronary heart disease of blood-stasis syndrome. Chin J Integr Med, 2008, 14: 267-273

15 李雪峰. 冠心病血疙证血小板差异功能蛋白的分析及药物干预研究. 博士学位论文. 北京: 中国中医科学院, 2010

16 Liu Y, Yin H J, Chen K J. Research on the correlation between platelet gelsolin and blood-stasis syndrome of coronary heart disease. Chin J Integr Med, 2011, 17: 587-592

17 Liu Y, Yin H J, Jiang Y R, et al. Correlation between platelet gelsolin level and different types of coronary heart disease. Chin Sci Bull, 2012, 57: $631-638$

18 Li G H, Shi Y, Chen Y, et al. Gelsolin regulates cardiac remodeling after myocardial infarction through DNase I -mediated apoptosis. Circ Res, 2009, 104: 896-904

19 刘玥, 蒋跃线, 殷惠军, 等. 凝溶胶蛋白与心血管疾病. 中国分子心脏病学杂志, 2011, 11: 50-53

20 Lee W M, Galbraith R M. The extracellular actin scavenger systern and actin toxicity. N Engl J Med, 1992, 326: 1335-1341

21 陈可冀, 李连达, 翁维良, 等. 血瘀证与活血化瘀研究. 中西医结合心脑血管病杂志, 2005, 1: 1-2

22 陈可冀. 活血化瘀方药降低心血管风险可能性的探索. 中国中西医结合杂志, 2008, 5: 389

23 Liu Y, Yin H J, Shi D Z, et al. Chinese herb and formulas of promoting blood circulation and removing blood stasis and antiplatelet therapies. Evid Based Compl Alt Med, 2012: 184503, doi: 10.1155/2012/184503

24 陆洁, 刘静, 申秀萍. 蛋白质组学在中药药理研究中的应用. 中药药理与临床, 2012, 28: 243-247

25 徐浩, 文川, 陈可冀, 等. 川药、赤莳及其有效部位配伍对载脂蛋白 $E$ 基因缺陷小鼠动脉弹样硬化斑块稳定性影响的研究. 中国中西医结 合杂志, 2007, 27: 513-518

26 李立志, 刘剑刚, 马鲁波, 等. 节苟胶囊对兔动脉粥样硬化模型脂质代谢及血小板聚集的影响. 中国中西医结合杂志, 2008, 28: 1100-1103

27 徐凤芹, 徐浩, 刘剑刚, 等. 芦苟胶囊对动脉弹样硬化兔血管平滑肌细胞增殖的影响. 中国中西医结合杂志, 2008, 28: 912-917

28 鹿小燕，史大卓，徐浩，等. 芎苟胶囊干预冠心病介入治疗后再狭窄的研究. 中国中西医结合杂志, 2006, 26: 13-17

29 Chen K J, Shi D Z, Xu H, et al. XS0601 reduces the incidence of restenosis: a prospective study of 335 patients undergoing percutaneous coronary intervention in China. Chin Med J (Engl), 2006, 119: 6-13

30 刘玥. 血小板骨架蛋白 gelsolin 在冠心病血瘀证中的作用及赤苟川芦有效组分的干预效应研究. 博士学位论文. 北京: 中国中医科学院, 2012 
31 Liu Y, Yin H J, Jiang Y R, et al. Correlation between platelet gelsolin and platelet activation level in acute myocardial infarction rats and intervention effect of effective components of Chuanxiong rhizome and Red peony root. Evid Based Complement Alternat Med, 2013: 985746, doi: $10.1155 / 2013 / 985746$

32 Yao Y, Wu W Y, Guan S H, et al. Proteomic analysis of differential protein expression in rat platelets treated with notoginsengnosides. Phytomedicine, 2008, 15: 800-807

33 Ma C, Yao Y, Yue Q X, et al. Differential proteomic analysis of platelets suggested possible signal cascades network in platelets treated with salvianolic acid B. PLoS ONE, 2011, 6: e14692

$34 \mathrm{Xu} \mathrm{H}$, Chen K J. Complementary and alternative medicine: is it possible to be mainstream? Chin J Integr Med, 2012, 18: 403-404

35 王台. 中医需要接受科学洗礼. 中国中西医结合杂志, 2012, 32: 1014-1022

36 谢鸣. “方证相关”逻辑命题及其意义. 北京中医药大学学报, 2003, 26: 11-12 